\title{
Detection, Characterization, and Bioavailability of Membrane-associated Iron in the Intact Sickle Red Cell
}

\author{
Takashi Sugihara, Tanya Repka, and Robert P. Hebbel \\ Department of Medicine, University of Minnesota Medical School, Minneapolis, Minnesota 55455
}

\begin{abstract}
It is hypothesized that membrane-associated iron in the sickle red cell is of pathophysiologic importance, but the actual existence of such iron in the intact cell has been questioned. Using a strategy whereby membrane iron can be detected through its bioavailability for catalyzing peroxidation, we used phospholipid exchange protein to load membranes of intact erythrocytes (RBC) with $\sim 2 \%$ phosphatidylethanolamine hydroperoxide (PEOOH) and monitored the development of peroxidation by-products during subsequent incubation. Normal RBC loaded with PEOOH developed very little peroxidation, but vitamin E-replete sickle RBC showed an exuberant peroxidation response that was not seen in cells loaded with control nonoxidized phosphatidylethanolamine. Ancillary studies of sickle $\mathrm{RBC}$ revealed that the catalytic iron included both heme iron and free iron located at the bilayer inner leaflet. Significantly, these studies also revealed that peroxidation after PEOOH loading is promoted by cellular dehydration and inhibited by hydration, thus identifying a dynamic interaction between hemoglobin (sickle $\gg$ normal) and membrane lipid. High-reticulocyte control RBC and sickle trait RBC behaved exactly like normal RBC, while $\mathrm{HbCC} \mathrm{RBC}$ and $\mathrm{RBC}$ having membranes gilded with hemoglobin iron because of prior exposure to acetylphenylhydrazine showed an abnormal peroxidation response like that of sickle $\mathrm{RBC}$. Indeed, the peroxidation response of $\mathrm{RBC}$ loaded with $\mathrm{PEOOH}$ paralleled amounts of iron measured on inside-out membranes prepared from them $(r$ $=0.783, P<0.01)$. These studies corroborate existence of membrane-associated heme and free iron in the intact sickle cell, and they document its bioavailability for participation in injurious peroxidative processes. That association of cytosolic sickle hemoglobin with membrane lipid is modulated by cell hydration status provides a mechanism that may help explain increased development of oxidative membrane lesions in abnormally dehydrated sickle $\mathrm{RBC}$ regardless of the mechanism underlying their formation. (J. Clin. Invest. 1992. 90:23272332.) Key words: phospholipid • peroxidation • heme • hemoglobin • oxidant
\end{abstract}

Address correspondence to Dr. Robert P. Hebbel, Box 480, University of Minnesota Hospital and Clinic, Harvard Street at East River Road, Minneapolis, MN 55455. Dr. Sugihara's present address is Department of Medicine, Kawasaki Medical School, Kurashiki, Japan.

Received for publication 5 March 1992 and in revised form 30 June 1992.

J. Clin. Invest.

(c) The American Society for Clinical Investigation, Inc.

$0021-9738 / 92 / 12 / 2327 / 06 \$ 2.00$

Volume 90, December 1992, 2327-2332

\section{Introduction}

In providing a permeability barrier for cell membranes, nature has used phospholipids that are partially unsaturated and has concomitantly employed a variety of protective mechanisms to limit the access of iron to lipid. Examples include the high affinity association of iron with various chelators, carriers, or storage proteins (e.g., heme, transferrin, ferritin). Nonetheless, the potential fragility of lipid in the presence of iron and oxygen predicts that opportunities for membrane damage could occur in nature. Indeed, it has been proposed that abnormalities of the sickle erythrocyte ( $\mathrm{RBC})^{1}$ illustrate the consequences of iron decompartmentalization $(1,2)$. The validity of this hypothesis, however, depends upon the key observation that the sickle membrane appears to be gilded with abnormal deposits of heme ${ }^{2}$ and nonheme iron (3-9). To reach this conclusion, however, investigators have always examined washed membranes derived from RBC that were disrupted by hypotonic lysis. It is disconcerting that the amount of measured membrane-associated hemoglobin, for example, diminishes as such washing proceeds. Thus, it has never been clear that the iron detected in such membrane preparations, even those obtained using washing of heroic proportions $(8,9)$, is truly representative of the state of the intact cell. Therefore, we devised a strategy to probe for existence of putative membrane-associated iron deposits. Since its biologic significance would lie in any ability to damage the membrane, we selected an approach that reflects the bioavailability of membrane-associated iron for stimulation of lipid peroxidation. The method uses phospholipid exchange proteins to load membranes of intact RBC with phospholipid hydroperoxide, with monitoring for the subsequent development of secondary (iron-dependent) peroxidation. Our results reveal the association of both heme iron and free iron with the sickle membrane's inner monolayer. They further identify a previously unsuspected dynamic interaction between hemoglobin and membrane lipid, a phenomenon that is greatly exaggerated for sickle compared to normal RBC.

\section{Methods}

Materials. We obtained $\left[{ }^{14} \mathrm{C}\right]$ phosphatidylcholine and $\left[{ }^{14} \mathrm{C}\right]$ triolein from New England Nuclear, Boston, MA; $\left[{ }^{14} \mathrm{C}\right]$ phosphatidyl-

1. Abbreviations used in this paper: IOM, inside-out membranes; $\mathrm{LOOH}$, lipid hydroperoxide; PC, phosphatidylcholine; PCOOH, PC hydroperoxide; PE, phosphatidylethanolamine; PEOOH, PE hydroperoxide; RBC, erythrocytes; TBARS, thiobarbituric acid reactive substances.

2. In this communication we use the term "heme" in the generic sense to include both free heme and various forms of hemoglobin, and we use the term "free iron" to represent nonheme iron that is also nonferritin iron, the convention used earlier $(8,9)$. 
ethanolamine from Amersham Corp., Arlington Heights, IL; and other reagents from Sigma Chemical Co., St. Louis, MO.

$R B C$ preparation. $\mathrm{RBC}$ were obtained from heparinized blood from normal donors, three patients with sickle cell anemia, a high reticulocyte control patient with hereditary spherocytosis, one individual with sickle trait, and a donor homozygous for $\mathrm{HbC}$. Each of the sickle donors used for the reported studies is known to have normal plasma and $\mathrm{RBC}$ membrane vitamin $\mathrm{E}$ contents. $\mathrm{RBC}$ were washed three times in buffer ( $10 \mathrm{mM}$ Hepes, $10 \mathrm{mM}$ glucose, $4 \mathrm{mM} \mathrm{KCl}, \mathrm{NaCl}$ to $290 \mathrm{mosmol} / \mathrm{liter}, \mathrm{pH} 7.4$ ) with removal of buffy coat. To create positive control samples, normal RBC at hematocrit $5 \%$ in this buffer were exposed to acetylphenylhydrazine $(0-10 \mathrm{mM})$ for $2 \mathrm{~h}$ at $37^{\circ} \mathrm{C}$. $\mathrm{RBC}$ were then thoroughly washed in the same buffer.

Loading $R B C$ with lipid hydroperoxide. Nonspecific phospholipid exchange protein was isolated from bovine liver exactly as previously described (10), and its activity was verified by measurement of mediated insertion of $\left[{ }^{14} \mathrm{C}\right]$ phosphatidylcholine (New England Nuclear) into RBC membranes.

For most of these experiments we used a synthetic phosphatidylethanolamine, 16:0,18:2 PE. To obtain PE hydroperoxide (PEOOH), 1 $\mathrm{mg} P E$ in methanol was admixed with rose bengal ( $125 \mu \mathrm{g})$ and photooxidized for $12 \mathrm{~h}$ (11). Comparison of such oxidized and control nonoxidized lipid preparations by thin layer chromatography (12) revealed identical and trivial amounts of lysolipid $(<1 \%)$ and free fatty acid $(<0.2 \%)$, moieties that are not transferred by the phospholipid exchange protein anyway (10). From a known mixture of PE and $\mathrm{PEOOH}$, we then made liposomes by sonication under nitrogen, on ice, and in Tris buffer $(90 \mathrm{mM} \mathrm{KCl}, 45 \mathrm{mM} \mathrm{NaCl}, 44 \mathrm{mM}$ sucrose, 10 $\mathrm{mM}$ Tris $/ \mathrm{HCl}, 0.1 \mathrm{mM}$ deferoxamine, $\mathrm{pH}$ 7.4) (13). The deferoxamine was included to minimize the potential problem of oxidative activation of hydroperoxide that might compromise the loading procedure itself. RBC at hematocrit (Hct) $30 \%$ were then admixed with liposomes ( $140 \mathrm{nmol}$ total PE + PEOOH/0.1 ml RBC) and phospholipid exchange protein ( $5 \mathrm{nmol} / 0.1 \mathrm{ml} \mathrm{RBC}$ ) in the deferoxamine-containing Tris buffer. After incubation at $37^{\circ} \mathrm{C}$ (usually for $60 \mathrm{~min}$ ), RBC were washed thoroughly using the deferoxamine-free Hepes buffer.

In control experiments (not shown), we incorporated trace amounts of $\left[{ }^{14} \mathrm{C}\right]$ triolein into the liposomes as a nonexchangeable marker of liposome location, and this showed that $<1 \%$ of lipid hydroperoxide newly associated with RBC could be accounted for by unincorporated lipid (i.e., by contamination with adherent liposomes). Observation of RBC shape after loading with PEOOH (or with nonoxidized PE) showed a significant degree of stomatocyte formation at zero-time and an increase therein over the course of the subsequent incubation; cells loaded with phosphatidylcholine hydroperoxide $(\mathrm{PCOOH})$ were echinocytic at zero-time, and this diminished during subsequent incubation.

Measures of peroxidation. RBC (either unmanipulated control, loaded with $\mathrm{PEOOH}$, or loaded with nonoxidized PE) were suspended to Hct $10 \%$ in the Hepes buffer (having no deferoxamine), and aliquots were taken before and after incubation for up to $3 \mathrm{~h}$ at $37^{\circ} \mathrm{C}$ for measurement of peroxidation parameters. Amount of lipid hydroperoxide was documented by iodometric assay, performed on extracted lipids (14). Generation of aldehydic peroxidation byproducts, as evidenced by formation of thiobarbituric acid reactive substances (TBARS), was monitored with correction for development of any non-TBARS chromogen as previously described (15). Immediately before these assays were performed, we added butylated hydroxytoluene and deferoxamine $(0.1 \mathrm{mM}$ each $)$ to preclude additional lipid decomposition during the assay itself. These measurements were made in duplicate, and duplicate values always varied by $<4 \%$ and usually by only $1 \%$.

Ancillary studies. We performed several studies using variations of the above methods. In one experiment we compared the effects of loading PEOOH versus PCOOH (made from 16:0,18:2 PC). In other studies, we replaced the soluble deferoxamine used during our standard PEOOH loading procedure with an amount of Sepharose-immobilized deferoxamine having the same iron binding capacity (kindly provided by Dr. Bo Hedlund, Biomedical Frontiers, Minneapolis, MN). In another experiment, we monitored TBARS formation by the mixture of 2 $\mathrm{mg} \mathrm{RBC}$ membrane protein (in the form of either intact RBC or insideout membranes prepared as described previously [8]) and $14 \mathrm{mg}$ phospholipid (in the form of liposomes composed of $2 \% \mathrm{PCOOH}$ and $98 \%$ $16: 0,18: 2 \mathrm{PC}$ ) in the absence of exchange protein. One experiment was performed using sickle RBC that were separated into top $10 \%$ and bottom $10 \%$ density subpopulations (16) and then loaded with PEOOH. Other experiments compared TBARS generation by PEOOH-loaded normal and sickle RBC suspended in deferoxaminecontaining Hepes buffer having 290,200, or 154, and 350 mosmol/ liter; control experiments (not shown) clearly documented that the amounts of RBC lysis occurring in these studies had no influence on amount of TBARS generation.

Membrane iron. To determine amounts of iron on washed RBC membranes in the conventional manner, we used the method that provides the cleanest possible RBC ghosts, that described by Kuross et al. (8). These were then converted to exhaustively washed inside-out membranes (IOM) exactly as described ( 8 ) and were used to determine amounts of membrane-associated heme (generic) and nonheme iron from absorbance at $398 \mathrm{~nm}$ in formic acid and from reactivity with ferrozine, respectively $(8,9)$.

Statistical analysis. Statistical comparisons used Student's $t$ test, paired or unpaired as appropriate.

\section{Results}

Sickle versus normal $R B C$. Fig. 1 depicts the spontaneous generation of TBARS from normal and sickle RBC incubated for 3 $h$, with and without prior loading with PEOOH or nonoxidized control PE. Amount of TBARS generation was trivial and virtually identical for normal RBC of all three types (loaded with $\mathrm{PEOOH}$, PE-loaded control, or unmanipulated control). In contrast, TBARS formation by sickle RBC of all three types was abnormal. This amount was virtually identical for unmanipulated sickle RBC and those loaded with PE, and in both cases this amount was two- to threefold greater $(P<0.001)$ than TBARS formation by normal RBC. Notably, sickle RBC loaded with PEOOH showed exuberant TBARS generation that was threefold greater $(P<0.001)$ than that from either type of control sickle $\mathrm{RBC}$ and that was sevenfold greater ( $P$ $<0.001$ ) than that from the PEOOH-loaded normal RBC.

Table I documents amounts of iron, both heme and non-
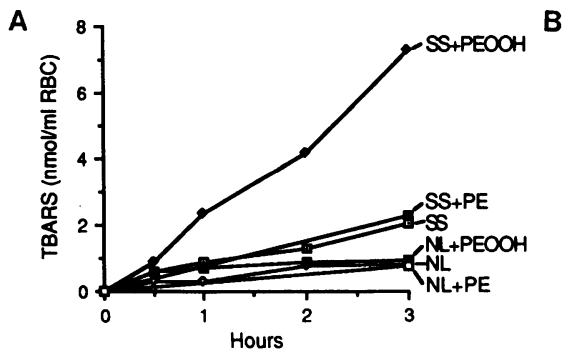

Figure 1. Peroxidation response of intact RBC loaded with PEOOH. Formation of TBARS was monitored for both normal and sickle RBC incubated with or without prior loading with $\mathrm{PEOOH}$ or nonoxidized PE. A representative experiment (loading both normal and sickle RBC with $2.5 \%$ $\mathrm{PEOOH}$ ) is shown in $A$. Data for three experiments (loading both normal and sickle RBC with $2.4 \pm 0.1 \%$ PEOOH) are summarized in $B$ (mean \pm SD for TBARS at $3 \mathrm{~h}$ ). 
Table I. Membrane Iron Content of RBC Used in These Studies

\begin{tabular}{lcc}
\hline \multicolumn{1}{c}{ RBC type } & Heme iron & Nonheme iron \\
\hline & $n m o l / m g$ & $n m o l / m g$ \\
Normal & $0.7 \pm 0.1$ & nd \\
Sickle & $1.6 \pm 0.2$ & $2.9 \pm 1.8$ \\
High reticulocyte & & \\
$\quad$ control & 0.8 & nd \\
Acetylphenylhydrazine & & \\
$\quad$ treated & & \\
$0 \mathrm{mM}$ & 0.7 & nd \\
$1 \mathrm{mM}$ & 1.4 & nd \\
$2.5 \mathrm{mM}$ & 1.6 & nd \\
$5.0 \mathrm{mM}$ & 2.8 & nd \\
$7.5 \mathrm{mM}$ & 8.5 & 0.8 \\
$10 \mathrm{mM}$ & 11.4 & 1.8 \\
& & \\
\hline
\end{tabular}

Heme and nonheme iron contents were determined on inside-out membranes prepared from exhaustively washed RBC ghost membranes $(8,9)$ and are expressed as nanomoles iron per milligram membrane protein. nd, not detectable (threshold of detection, 0.05 $\mathrm{nmol} / \mathrm{mg})$.

heme, measured in the extensively washed IOM prepared from the RBC used in these studies. These values correspond well with those previously observed for normal and sickle RBC $(8,9)$.

Positive and negative control experiments. Sickle RBC samples are typically enriched in young $\mathrm{RBC}$, so we examined a high reticulocyte $(15 \%)$ control sample from a patient with hereditary spherocytosis. This sample had only normal amounts of IOM iron (Table I), and when PEOOH-loaded it showed no increased tendency to form TBARS (data not shown).

In contrast, positive control RBC treated with up to $10 \mathrm{mM}$ acetylphenylhydrazine were strikingly abnormal. As measured immediately after this treatment, they had elevated amounts of iron on their IOM (Table I), and they showed greatly exaggerated TBARS generation in response to PEOOH loading and subsequent incubation (Fig. 2). Indeed, we found a strong correlation between TBARS generation and IOM total iron for acetylphenyl-hydrazine-treated cells $(r=0.817, P<0.05)$ and

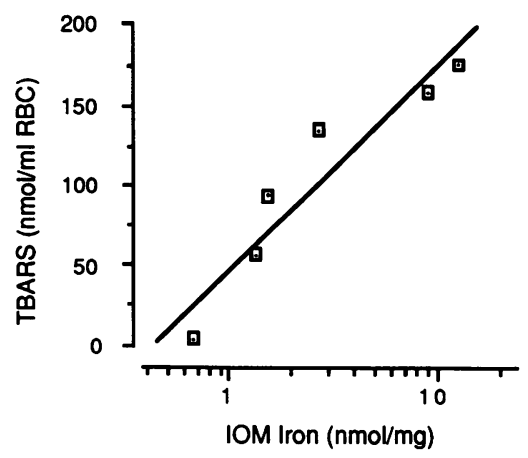

Figure 2. Peroxidation response of acetylphenylhydrazine-treated RBC. Normal RBC were exposed to $0,1,2.5,5,7.5$, and $10 \mathrm{mM}$ acetylphenylhydrazine, and TBARS response was observed during $3 \mathrm{~h}$ of incubation following loading with $2.3 \%$ PEOOH. Results are shown as a function of the total iron (heme

plus nonheme) measured in IOM prepared from these cells (from Table I). for RBC samples of all types used in these experiments $(r$ $=0.783, P<0.01$ ).

Validating control experiments. A variety of other control experiments were done to validate the usefulness of the lipid hydroperoxide $(\mathrm{LOOH})$ loading technique used in these studies. $\mathrm{RBC}$ subjected to a sham loading procedure (i.e., incubated with PEOOH liposomes but without exchange protein) remained identical to the unmanipulated control cells and did not develop the peroxidation changes induced by $\mathrm{PEOOH}$ loading (data not shown).

By performing organic extractions of separated membranes and cytosol, we documented that both $\left[{ }^{14} \mathrm{C}\right] \mathrm{PEOOH}$ and $\left[{ }^{14} \mathrm{C}\right]-$ PCOOH loaded into RBC membranes remains entirely within the cell membrane over at least the 3-h period of these experiments (data not shown). Likewise, when RBC loaded with either $\mathrm{PEOOH}$ or $\mathrm{PCOOH}$ were incubated in presence of 0.1 $\mathrm{mM}$ butylated hydroxytoluene (to prevent catalysis of detectable peroxidation [13]), amount of measured membrane $\mathrm{LOOH}$ was found to be perfectly stable over $3 \mathrm{~h}$ (data not shown), indicating an absence of significant $\mathrm{LOOH}$ repair at least over the period of these experiments.

Liposomes containing 50\% LOOH that were incubated by themselves for $3 \mathrm{~h}$ in the iron-free buffer used for these studies were perfectly stable and showed no spontaneous peroxidation whatsoever; however, if $10 \mu \mathrm{M}$ iron (as $\mathrm{Fe}^{2+} / \mathrm{ADP}$ in a 1:2 molar ratio) was deliberately added, they then developed a peroxidation response (data not shown). Notably, liposomes containing either $\mathrm{PEOOH}$ or $\mathrm{PCOOH}$ were stimulated by precisely the same degree in this regard, indicating equivalent activation of the two LOOH types by iron (data not shown).

Ancillary studies of sickle $R B C$. To identify which sickle membrane iron compartment(s) provided catalytic iron in the above experiments, we performed several ancillary studies.

We wished to estimate relative contributions of heme iron and free (i.e., chelatable) iron to this catalytic ability. For this we modified our standard procedure in three experiments so that sickle RBC were loaded with PEOOH $(2.2 \pm 0.3 \%)$ in the presence of Sepharose-immobilized deferoxamine rather than soluble deferoxamine (to preserve our original intention of chelating any iron-contaminating buffers but to avoid any possibility of chelating intracellular iron ). In each experiment, the PEOOH-loaded cells were then incubated in one of three ways: with soluble deferoxamine, with immobilized deferoxamine, or without any chelator. Those incubated with immobilized chelator manifested identical TBARS formation to those incubated with no chelator, while those incubated with soluble chelator showed $35.0 \pm 3.6 \%$ lower TBARS formation $(P<0.01)$. This reveals a significant contribution from free iron as well as from heme iron, and it provides evidence that the catalytic free iron is associated with the inner monolayer.

In an effort to further establish that catalytic iron is associated with the inner rather than the outer monolayer, we performed two types of experiments. In one, we monitored TBARS formation from sickle RBC loaded in parallel with either $\mathrm{PEOOH}$ or $\mathrm{PCOOH}$. The peroxidation response was markedly greater for those loaded with PEOOH (Fig. 3). In the second approach, we examined the ability of the sickle RBC membrane in two different forms to catalyze peroxidation of liposomal lipid when admixed with PC/PCOOH liposomes. Negligible new $\mathrm{LOOH}$ formation resulted when the liposomes were exposed to intact RBC, consistent with sequestration of catalytic iron within the cell, but brisk $\mathrm{LOOH}$ formation was 


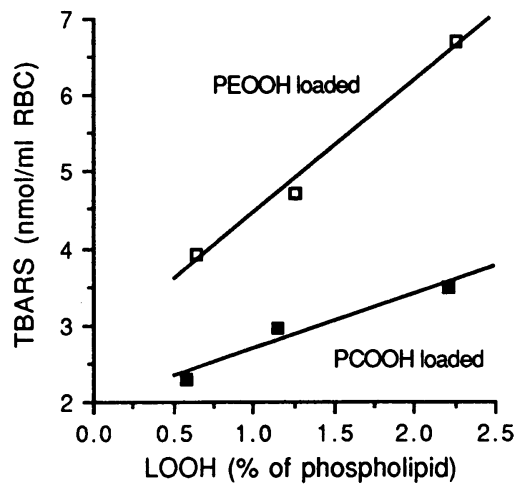

seen when liposomes were exposed to the IOM prepared from these same sickle RBC (Fig. 4).

Finally, we examined RBC for potential interaction between cytosolic $\mathrm{Hb}$ and the lipid bilayer. We aliquoted PEOOH-loaded normal and sickle RBC into Hepes buffers having adjusted osmolalities ( 154 or 200,290 , and 350 mos$\mathrm{mol} /$ liter) and monitored their TBARS response over $3 \mathrm{~h}$. In this case, because we were interested in the influence of hemoglobin only, we included deferoxamine in all buffers to eliminate the effect identified above for membrane free iron. Fig. 5 shows both a single experiment and data summarizing three experiments demonstrating that peroxidation response was proportional to buffer osmolality. Compared to RBC at 290 mosmol/liter, those in hypotonic buffer had significantly less TBARS formation ( $P=0.018$ for normal and $P=0.006$ for sickle), and those in hypertonic buffer had significantly greater TBARS formation $(P=0.021$ for normal and $P=0.033$ for sickle). Although this was true for both normal and sickle $\mathrm{RBC}$, the effect was greatly exaggerated for the HbS-containing cells. For any given buffer osmolality, TBARS generation by sickle $\mathrm{RBC}$ greatly exceeded that of normal $\mathrm{RBC}(P<0.001$ in each case). $R B C$ that were transferred from hypotonic to isotonic buffer part way into the incubation period promptly lost the protective benefit of the hypotonic conditions and nearly doubled their peroxidation rate for the remainder of the experiment (Fig. 5, $A$ and $B$ ). It should be noted that control experiments in a model system (RBC ghosts treated with the peroxidant t-butylhydroperoxide) documented that, after it is initiated, peroxidation rate and extent are not influenced at all by buffer osmolality (data not shown). Thus the observed effect of

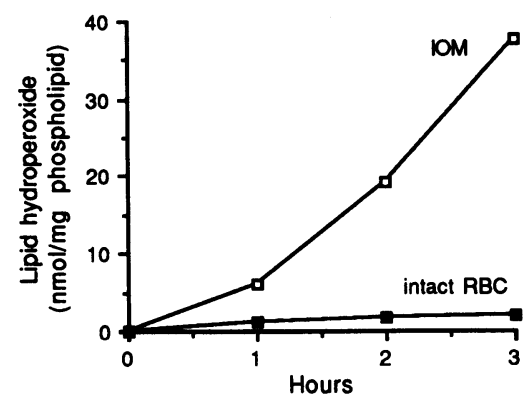

Figure 4. Catalytic iron resides on the inner membrane surface. PC/ $\mathrm{PCOOH}$ liposomes (in the absence of phospholipid exchange protein) were admixed with sickle RBC membranes in the form of either intact RBC or inside-out membranes. The peroxidation response (mea-

sured as $\mathrm{LOOH}$ formation) indicates contact of catalytic iron with liposomal lipid. A representative experiment (one of two) is shown.
A

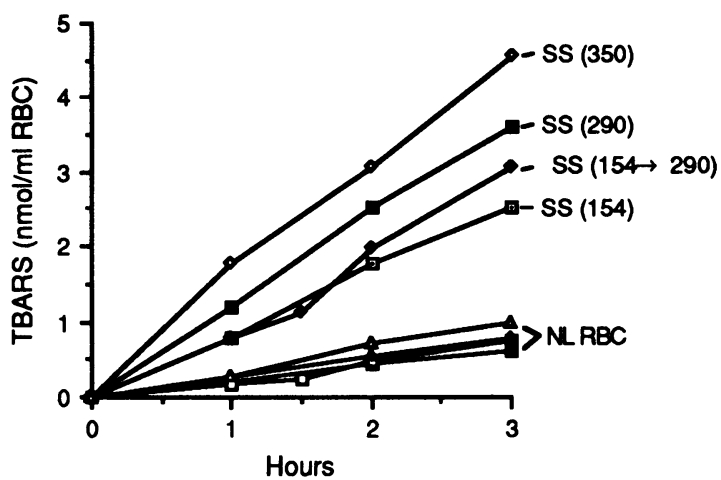

B

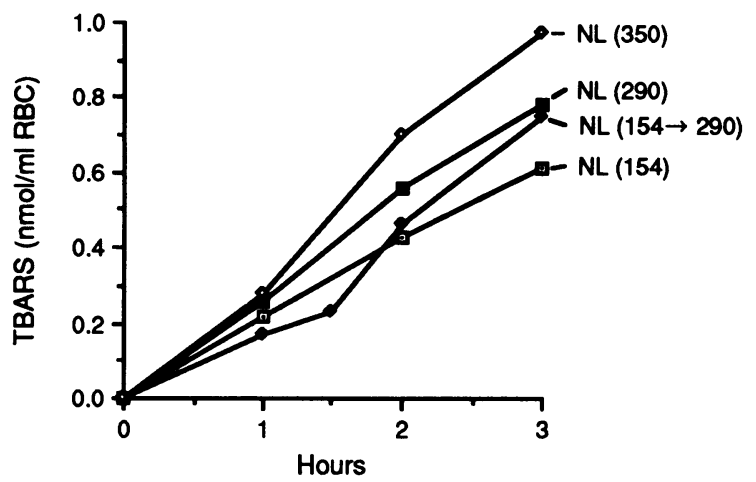

C

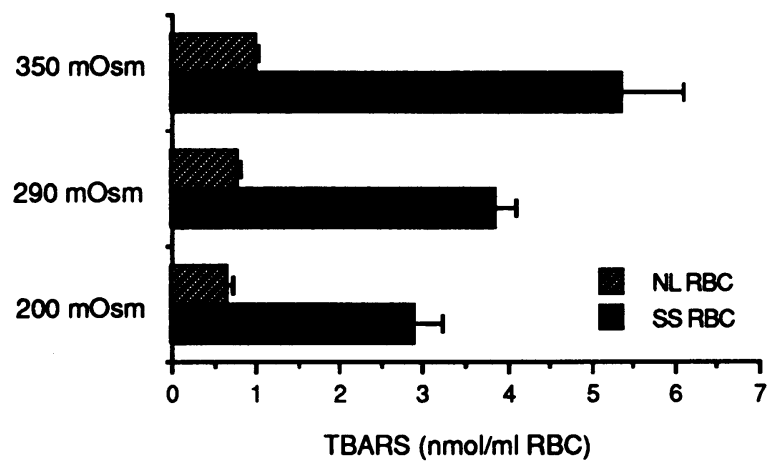

Figure 5. Cellular hydration status influences peroxidation response. Sickle and normal RBC were loaded with PEOOH, and TBARS formation (in presence of deferoxamine) was monitored during incubation in buffers having varying osmolality as indicated in parentheses (mosmol/liter). Representative experiments are shown for sickle RBC loaded with $2.0 \%$ PEOOH $(A)$ and normal $\mathrm{RBC}$ loaded with $1.9 \% \mathrm{PEOOH}(A$ and $B$ ). In each case, one aliquot of $\mathrm{RBC}$ was transferred from hypotonic to isotonic buffer after $1.5 \mathrm{~h}$ (curve labeled $154 \rightarrow 290$ ). Data for three additional experiments on normal and sickle RBC (loaded with $2.1 \pm 0.2$ and $2.1 \pm 0.1 \% \mathrm{PEOOH}$, respectively) are shown in $C$ (mean \pm SD for TBARS at $3 \mathrm{~h})$.

osmolality (Fig. 5) reflects differences in its initiation, i.e., availability of catalytic iron.

Since these latter studies suggest that there is an abnormal but dynamic interaction between cytosolic $\mathrm{HbS}$ and the membrane that is influenced by cell hydration state, we also per- 
formed two additional experiments. First, we obtained results consistent with those immediately above by density separating sickle RBC into top- $10 \%$ and bottom-10\% fractions, loading these RBC with PEOOH, and monitoring TBARS generation. The well-hydrated, least dense cells showed no improvement at $200 \mathrm{mosmol} /$ liter compared with $290 \mathrm{mosmol} / \mathrm{liter}$; but the most dense, dehydrated RBC did show significant improvement (Fig. 6). In the second experiment, we examined cells containing another mutant $\mathrm{Hb}$ that is believed to interact abnormally with the RBC membrane, HbC. These cells also showed a somewhat exaggerated TBARS response to PEOOH loading, with marked exacerbation under hypertonic conditions (evident by comparing data in Table II with those in Fig. 1). In contrast to these results, in one experiment we found that sickle trait RBC loaded with PEOOH were indistinguishable from normal RBC at either 290 or 350 mosmol.

\section{Discussion}

These experiments establish the existence of abnormal deposits of iron in association with the sickle RBC membrane. By examining intact $R B C$, we have avoided potential sources of artifact such as catastrophic membrane disruption and exposure to hypotonic solutions used in previous studies on washed membranes (3-9). Rather, we inserted PEOOH into the membrane of intact cells, a strategy that can be used because inserted phospholipid remains constrained within the lipid bilayer and because lipid hydroperoxide is stable in the absence of transition metals (as shown in validating control experiments). Hence, initiation of peroxidation is strictly dependent upon activation of the loaded hydroperoxide through interaction with membrane-associated iron. The induced TBARS response must reflect the further peroxidation of endogenous membrane phospholipid since the phospholipid probe molecule used for these studies has only two double bonds and itself cannot support significant TBARS formation. Therefore, the exaggerated peroxidation response of sickle $\mathrm{RBC}$ observed in these studies can be taken as direct evidence for presence of abnormal deposits of iron on membranes of the intact sickle cells.

The stomatocytic shape change manifested by these loaded cells implies that the introduced $\mathrm{PEOOH}$ translocates to the inner monolayer, but formal proof of $\mathrm{LOOH}$ transbilayer

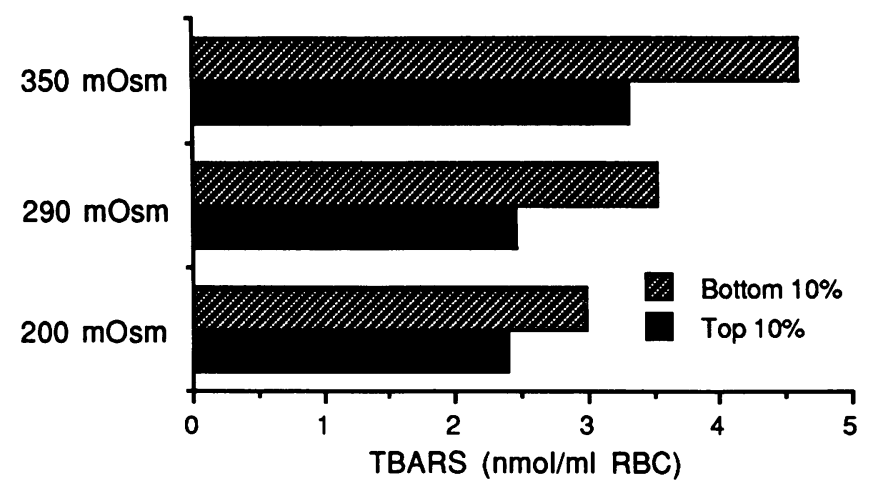

Figure 6. Hypotonic medium protects most dense sickle RBC. Sickle RBC were separated by density into top- $10 \%$ and bottom- $10 \%$ fractions. These were PEOOH loaded ( 2.2 and $2.4 \%$ PEOOH, respectively) and examined for peroxidation response (shown as TBARS over $3 \mathrm{~h}$ ) during incubation in media of different osmolalities as indicated.
Table II. TBARS Response of HbCC RBC

\begin{tabular}{lccc}
\hline & & \multicolumn{2}{c}{ TBARS } \\
\cline { 3 - 4 } RBC type & $\begin{array}{c}\text { PEOOH } \\
\text { loaded }\end{array}$ & 290 mosmol & 350 mosmol \\
\hline & $\%$ & \multicolumn{2}{c}{$n m o l / m l ~ R B C / 3 h$} \\
Experiment 1 & & & \\
HbCC & 1.9 & 0.95 & 1.95 \\
HbAA & 1.8 & 0.65 & 0.86 \\
Experiment 2 & & & \\
HbCC & 2.1 & 1.20 & 2.69 \\
HbAA & 2.2 & 0.98 & 1.14 \\
\hline
\end{tabular}

Intact $\mathrm{HbCC} \mathrm{RBC}$ were loaded with $\mathrm{PEOOH}$, and TBARS generation was measured during subsequent incubation in buffer having either 290 or $350 \mathrm{mosmol} /$ liter.

movement has not been attempted. Nevertheless, our results necessarily identify iron having some degree of intimate contact with membrane lipid, presumably at the inner monolayer in sickle membranes. Our observation of a much greater peroxidation response initiated in sickle $\mathrm{RBC}$ by the inner leaflet lipid ( $\mathrm{PEOOH})$ compared to the outer leaflet lipid $(\mathrm{PCOOH})$ is consistent with this but does not definitively prove it. More convincing is our demonstration that brisk peroxidation of exogenous lipid (in form of liposomes) occurs when the liposomes are exposed to the inner surface of sickle membranes (i.e., IOM) but not when they are exposed to the outer surface. Thus, in aggregate, our results strongly support the inner bilayer association of iron in sickle membranes.

Regarding the type of iron on sickle membranes, the present data corroborate previous deductions based on less direct evidence $(1,2,8,9)$. Kuross found that nonheme iron is removed from sickle IOM only by treatment with phospholipase, implying an association of free iron with the polar head groups of inner leaflet aminophospholipid (9). Our present results confirm association of free iron (i.e., nonheme iron chelatable with deferoxamine) with the inner aspect of the membrane in the intact sickle RBC. Indeed, $\sim 35 \%$ of the catalytic activity manifested by sickle membranes is provided by iron of this type. It also is likely that membrane-associated hemoglobin is in abnormal proximity to lipid. Studies of washed RBC ghosts previously suggested presence of denatured hemoglobin on sickle membranes (3-8). Consistent with this, our results show that iron compounds not chelatable by deferoxamine provide over half the detected catalytic activity. This probably represents hemoglobin iron since the amount of free heme in these membranes is so low $(8,9)$, although it is true that the actual location of this free heme (which is not yet known), rather than its amount, would be the major determinant of its catalytic activity. Catalytic hemoglobin iron could be in the form of deposits of denatured hemoglobin on the membrane or in the form of soluble (cytosolic) hemoglobin in abnormally close proximity to membrane lipid. The latter possibility was suggested by the studies of Eisinger et al. (17) using fluorescence quenching to show excessive concentrations of heme at the cytosolic/membrane interface of intact sickle RBC. ${ }^{3}$ That this

3. Eisinger's contrasting failure to detect membrane hemoglobin in sickle ghosts using that technology (17) may have reflected the predict- 
represents cytosolic hemoglobin rather than an irreversible deposition of denatured hemoglobin was supported by a diminution of quenching when the sickle cells were deoxygenated (17).

Thus, it is particularly intriguing that our data suggest at least some portion of the catalytic activity ascribable to hemoglobin iron, in fact, derives from cytoplasmic hemoglobin. The evidence for this is that catalysis of peroxidation in $\mathrm{PEOOH}-$ loaded RBC is significantly influenced by cell hydration status, with the elevated cytosolic hemoglobin concentration that accompanies dehydration being harmful and improved hydration being protective. Our study of density-separated sickle RBC subpopulations provides supporting evidence for this interpretation, as does our examination of $\mathrm{RBC}$ containing $\mathrm{HbC}$, another mutant that is believed to interact abnormally with the $\mathrm{RBC}$ membrane. This hydration effect reflects a difference in amount of catalytic iron available for interaction with $\mathrm{PEOOH}$ since our control studies documented that after its initiation, membrane peroxidation proceeds to the same extent regardless of medium osmolality. Of particular significance, the influence of cell hydration state on TBARS response is greatly exaggerated for sickle compared to normal RBC. In fact, Eisinger's data also are consistent with the notion that $\mathrm{RBC}$ dehydration has a markedly abnormal effect on heme concentration at the membrane/cytosolic interface for sickle RBC compared to normal (17). The normalcy we observed for sickle trait cells is consistent with the fact that their cytoplasmic $\mathrm{HbS}$ concentration is much lower than that of HbSS RBC (and with the fact that they do not have abnormal iron deposits on their membranes $[8,9])$. Notably, previous studies documented that cell hydration state governs a unique dynamic interaction between $\mathrm{HbS}$ and the membrane cytoskeleton, as evidenced by an influence exerted on microrheologic properties of $\mathrm{RBC}$ membranes $(18,19)$. Remarkably, the present data suggest that this behavior of $\mathrm{HbS}$ extends to a reversible association with membrane lipid as well. This phenomenon will require further, detailed study, but these initial observations provide a tantalizing insight into a previously unsuspected aspect of the cytosol $/ \mathrm{mem}$ brane interface.

Significantly, the amount of peroxidation induced by PEOOH loading correlated strongly with the quantity of iron measured in extensively washed IOM prepared from these $\mathrm{RBC}$, lending support to the belief that studies of disrupted membranes can be informative if those membranes are suitably prepared (i.e., turned inside-out and extensively washed) (8). It is conceivable that refinement of the present method might be useful as a measure of membrane iron content, but this would at best provide only semi-quantitative data since the endpoint clearly is influenced by other factors, such as membrane vitamin $\mathrm{E}$ content. The sickle samples used herein were specifically chosen from patients known to have normal membrane vitamin E content.

Thus, the present studies indicate that there is abnormal and intimate association of iron (both heme and nonheme) with the membrane of the intact sickle $\mathrm{RBC}$ and that this iron is bioavailable for participation in peroxidative biochemistry.

able artifactual diminution of signal quenching resulting from depletion of probe molecules in that portion of the lipid bilayer closest to the membrane-bound hemichrome because of its association with clustered band 3 molecules $(2)$.
This may be relevant to development of sickle membrane defects insofar as these derive from pathologic oxidation $(1,2)$. Notably, the belief that development of oxidative membrane defects precedes (and perhaps causes) formation of dehydrated cells must now be reexamined since the ability of cell hydration status to reversibly modulate hemoglobin association with lipid provides a possible mechanism for accelerated development of oxidative membrane lesions in the most dehydrated sickle cells, regardless of the mechanism underlying their formation. Not surprisingly, the peroxidation occurring during use of the present experimental model is accompanied by thiol oxidation (data not shown ); whether a similar process underlies development of the membrane thiol oxidation observed in unmanipulated sickle $\operatorname{RBC}(1,2)$ remains to be seen.

\section{Acknowledgments}

We thank Carol Taubert for invaluable assistance.

This work was supported by the National Institutes of Health (grants HL-30160 and HL-37528).

\section{References}

1. Hebbel, R. P. 1990. The sickle erythrocyte in double jeopardy: autoxidation and iron decompartmentalization. Sem. Hematol. 27:51-69.

2. Hebbel, R. P. 1991. Beyond hemoglobin polymerization: the red blood cell membrane and sickle disease pathophysiology. Blood. 77:214-237.

3. Asakura, T., K. Minakata, K. Adachi, M. O. Russell, and E. Schwartz. 1977. Denatured hemoglobin in sickle erythrocytes. J. Clin. Invest. 59:633-640.

4. Kim, H. C., S. Friedman, T. Asakura, and E. Schwartz. 1980. Inclusions in red blood cells containing HbS or HbC. Br. J. Haematol. 44:547-554.

5. Campwala, H. Q., and J. F. Desforges. 1982. Membrane-bound hemichrome in density-separated cohorts of normal (AA) and sickled (SS) cells. $J$. Lab. Clin. Med. 99:25-28.

6. Sears, D. A., and M. G. Luthra. 1983. Membrane-bound hemoglobin in the erythrocytes of sickle cell anemia. J. Lab. Clin. Med. 102:694-698.

7. Rice-Evans, C., S. C. Omorphos, and E. Baysal. 1986. Sickle cell membranes and oxidative damage. Biochem. J. 237:265-269.

8. Kuross, S. A., B. H. Rank, and R. P. Hebbel. 1988. Excess heme in sickle erythrocyte inside-out membranes: possible role in thiol oxidation. Blood. 71:876-882.

9. Kuross, S. A., and R. P. Hebbel. 1988. Nonheme iron in sickle erythrocyte membranes: association with phospholipids and potential role in lipid peroxidation. Blood. 72:1278-1285.

10. Bloj, B., and D. B. Zilversmit. 1983. Nonspecific lipid transfer protein from rat and beef liver: purification and properties. Methods Enzymol. 98:574581 .

11. van Kuijk, F. J. G. M., D. W. Thomas, R. J. Stephens, and E. A. Dratz. 1985. Gas chromatography_mass spectrometry method for determination of phospholipid peroxides. I. Transesterification to form methyl esters. J. Free Radicals Biol. Med. 1:215-225.

12. Skipski, V.P., R. F. Peterson, and M. Barclay. 1964. Quantitative analysis of phospholipids by thin-layer chromatography. Biochem. J. 90:374-378.

13. Sugihara, T., W. Rawicz, E. A. Evans, and R. P. Hebbel. 1991. Lipid hydroperoxides permit deformation-dependent leak of monovalent cation from erythrocytes. Blood. 77:2757-2763.

14. Aust, S. D. 1985. Lipid peroxidation. In CRC Handbook of Methods for Oxygen Radical Research. R. A. Greenwald, editor. CRC Press, Boca Raton, FL. 203-207.

15. Gilbert, H. S., D. D. Stump, and E. F. Roth, Jr. 1984. A method to correct for errors caused by generation of interfering compounds during erythrocyte lipid peroxidation. Anal. Biochem. 137:282-286.

16. Murphy, J. R. 1973. Influence of temperature and method of centrifugation on the separation of erythrocytes. J. Lab. Clin. Med. 82:334-341.

17. Eisinger, J., J. Flores, and R. M. Bookchin. 1984. The cytosol-membrane interface of normal and sickle erythrocytes. J. Biol. Chem. 259:7169-7177.

18. Evans, E. A., and N. Mohandas. 1987. Membrane-associated sickle hemoglobin: a major determinant of sickle erythrocyte rigidity. Blood. 70:1443-1449.

19. Evans, E., N. Mohandas, and A. Leung. 1984. Static and dynamic rigidities of normal and sickle erythrocytes. Major influence of cell hemoglobin concentration. J. Clin. Invest. 73:477-488. 